There is a high mobility rate in the US population; between $1987-1988$ over $17 \%$ of the population moved house and more than $6 \%$ moved to a different state. Taking into account that they do not have the equivalent of a general practitioner as found in the UK, follow-up of patients becomes very difficult. This results in duplication of work, delays in treatment while trying to collect relevant data, and difficulties monitoring type and extent of care offered to all patients.

Self referral is the norm in the USA, allowing patients much more input into what type of specialist they see, how long they attend and when they decide to re-refer themselves to someone else. Obviously difficulties in transference may not be addressed but avoided, and may hinder treatment regimes. The decision to attend two therapists of diametrically opposing views, either in succession or concurrently, may lead to obvious difficulties in treatment, for patient and therapists.

Patient autonomy is also more in evidence in the US setting. Anorexic patients, for example, may not consent to bed rest or increasing calorie consumption and so treatment plans will have to accommodate this. Insurance companies also influence the hospital treatment a professional may wish to prescribe by determining length of stay and types of treatment which will be reimbursed.

This covert but powerful influence accounts for many of the differences in attitudes to medical care that exist between the US and UK systems. In the final analysis, cost containment must be balanced with ultimate care to all patients to ensure adequacy of service provided.

13C Vanbrugh Hill

Blackheath, London SE3 TUE

References are available on request to Dr McNicholas.

\section{Smoking among psychiatric in-patients}

DeAR SiRS

I read with interest the article 'Smoking Among Psychiatric In-patients' by Claudia Corby and Jennifer Barraclough (Psychiatric Bulletin, June 1992, 16, 235-236). They did not report the frequency of smoking among those patients detained under a section of the Mental Health Act 1983. As hospitals move towards non-smoking or restricted smoking policies the rights of this particularly vulnerable group of psychiatric patients should be considered. No-one has suggested that non-psychiatric patients should compulsorily be made to give up smoking (Lavin, 1990) and the same should be true for psychiatric patients. In contrast with physical illnesses, the restriction of smoking is not likely to facilitate recovery, indeed for heavy smokers it might even constitute an additional emotional stress.

When non-smoking policies are drawn up for psychiatric hospitals, it should be taken into account that detained patients cannot exercise their right to leave the premises. For example, in one hospital at which I worked, the sale of cigarettes in the hospital shop was stopped but arrangements were made for staff to purchase cigarettes for those patients detained under the Mental Health Act who requested them.

There is no doubt that smoking is injurious to health. Where patients express a wish to reduce their smoking they should be encouraged and supported. Non-smoking areas on wards certainly should be made as attractive as possible. It should not be forgotten that detained patients are the illest group and that their needs should be given special consideration.

Queen Elizabeth Psychiatric Hospital

Mindelsohn Way, Birmingham B15

A. K. Stanley

\section{Reference}

Lavin, M. (1990) Let the patients smoke: a defence of a patient privilege. Journal of Medical Ethics, 16, 136-140.

\section{Patients too intoxicated for assessment}

DeAr Sirs

We read with concern a letter from Huw Thomas (Psychiatric Bulletin, June 1992, 16, 368) regarding patients who are too intoxicated for assessment and his extraordinary proposed solution to breathalyse patients on arrival, presumably to exclude them from being assessed. We believe that even the drinkers have the right of assessment and that psychiatrists have the duty to do so. Many of these patients have other psychiatric and medical problems unrelated to drinking.

A probable reason for the low rate of subsequent uptake in some areas may be the hostile approach of those who assess intoxicated patients. An empathetic approach which respects the dignity of the patient is the obligation of medical practitioners and may lead to a better outcome. Dr Thomas is asking for longterm solutions for this problem. If the treatment approach is community based, with involvement and support of families and availability of home detoxification, more problem drinkers could be helped and, if part of community domiciliary orientated intervention, may lead to reduction of numbers of people coming to the wards for help while intoxicated.

BASEM FARID

Leicestershire Community Alcohol Team

DOUglas CAMERon

Drury House, 50 Leicester Road

Narborough, Leicester LE9 5DF 